\title{
Urinary System Findings Sponsor-Defined Identifier
}

National Cancer Institute

\section{Source}

National Cancer Institute. Urinary System Findings Sponsor-Defined Identifier. NCI

Thesaurus. Code C162200.

One or more sponsor defined characters used to identify, name, or characterize the urinary system finding. 